\title{
Photon kinetics in plasmas
}

\author{
V.G. Morozov ${ }^{1}$, G. Röpke² \\ ${ }^{1}$ Moscow State Institute of Radioengineering, Electronics, and Automation (Technical University), Vernadsky \\ Prospect, 78, 119454 Moscow, Russia \\ 2 Universität Rostock, Institut für Physik, D-18051 Rostock, Germany
}

Received June 24, 2009

\begin{abstract}
We present a kinetic theory of radiative processes in many-component plasmas with relativistic electrons and nonrelativistic heavy particles. Using the non-equilibrium Green's function technique in many-particle QED, we show that the transverse field correlation functions can be naturally decomposed into sharply peaked (nonLorentzian) parts that describe resonant (propagating) photons and off-shell parts corresponding to virtual photons in the medium. Analogous decompositions are obtained for the longitudinal field correlation functions and the correlation functions of relativistic electrons. We derive a kinetic equation for the resonant photons with a finite spectral width and show that the off-shell parts of the particle and field correlation functions are essential to calculate the local radiating power in plasmas and recover the results of vacuum QED. The plasma effects on radiative processes are discussed.
\end{abstract}

Key words: many-particle QED, nonequilibrium Green's functions, relativistic plasmas

PACS: $52.20 .-j, 52.25 \mathrm{Dg}, 52.38 \mathrm{Ph}, 52.250 \mathrm{~s}, 52.27 \mathrm{Ny}$

\section{Introduction}

It was about sixty years ago that N.N. Bogolyubov developed a method for deriving kinetic equations more general than the Boltzmann equation or its simplest modifications. His famous monograph [1] considerably affected the further evolution of kinetic theory. At present, the most highly developed methods for treating kinetic processes in many-particle systems from first principles are the density matrix method [2-4] and the real-time Green's function method [5-7]. Of the two methods, the former can be viewed as a direct extension of Bogolyubov's approach. Although the Green's function method seems to start from quite different ideas, it should be noted that some essential ingredients of this method are closely related to the fundamental Bogolyubov's boundary condition of weakening of initial correlations [7,8].

In this talk based on our recent work [9], we present a general kinetic framework for studying radiative processes in many-component plasmas with relativistic electrons. The interest to the theory of nonequilibrium relativistic plasmas has been regenerated by current developments in ultra-short high-intensity laser technology and recent progress in laser-plasma experiments [10]. Radiative processes contribute to stopping power for highly relativistic electron beams in laserplasma systems, and they present interest as an example of fundamental QED processes in a medium [11]. Note also that the measurement of angular distribution of $\gamma$ rays in laser-plasma experiments has been found to be a powerful diagnostic tool [12].

A systematic approach to nonequilibrium QED plasmas based on the Green's function technique was developed by Bezzerides and DuBois [13]. Within the weak coupling approximation, they derived a covariant particle kinetic equation involving electron-electron collisions and Cherenkov emission and absorption of plasmons. Note, however, that the kinetics of transverse photons and radiative phenomena are mainly related to higher-order processes, like bremsstrahlung or Compton scattering. Although these processes have been studied in great detail within the framework of vacuum QED, they have not been studied to sufficient generality and clarity in many-particle nonequilibrium QED when plasma effects are of importance. It is our purpose to perform the still 
missing formulation of photon kinetics which includes relativistic and plasma effects, and, where appropriate, recovers the results of vacuum QED.

For the problems under consideration, there is a preferred frame of reference, the rest frame of the system, in which heavy particles (protons and ions) are nonrelativistic. It is thus convenient to choose the Coulomb gauge, allowing a description of electromagnetic fluctuations in terms of longitudinal and transverse (photon) modes. Throughout the paper we use the system of units with $c=\hbar=1$ and the Heaviside's units for electromagnetic field, i. e., the Coulomb force is written as $q q^{\prime} / 4 \pi r^{2}$. Although we work in the Coulomb gauge and in the rest frame of the system, many formulas are represented more compactly in the relativistic four-notation. The signature of the metric tensor $g^{\mu \nu}$ is $(+,-,-,-)$. Summation over repeated Lorentz (Greek) and space (Latin) indices is understandable. We use the usual abbreviation $\not a=\gamma^{\mu} a_{\mu}$ for any four-vector $a^{\mu}$. Our convention for the matrix Green's functions on the time-loop Schwinger-Keldysh contour follows Botermans and Malfliet [7].

\section{Functional description}

The dynamics of particles and electromagnetic fluctuations in a plasma can be described by using the following formal device [13]. Let us assume that the system was perturbed from its initial state by some prescribed $c$-number external four-current $J^{(\text {ext })} \mu(\mathbf{r}, t)=\left(\varrho^{\text {(ext) }}, \mathbf{J}^{\text {(ext) }}\right)$, where $\varrho^{(\mathrm{ext})}(\mathbf{r}, t)$ is the external charge density and $\mathbf{J}^{(\mathrm{ext})}(\mathbf{r}, t)$ is the external current density. The plasma is assumed to be weakly coupled, so that the effects of initial correlations die out after a few collisions. In this case the Bogolyubov condition of complete weakening of initial correlation may be used, i. e., all ensemble averages may be calculated at $t \rightarrow-\infty$ with a statistical operator $\varrho_{0}$ admitting Wick's decomposition. The basic quantities are the field and particle Green's functions defined on the time-loop Schwinger-Keldysh contour $C$ which runs from $-\infty$ to $+\infty$ along the chronological branch $C_{+}$and then backwards along the antichronological branch $C_{-}$[7]. From now on, the underlined variables $\underline{k}=\left(\underline{t}_{k}, \mathbf{r}_{k}\right)$ indicate that $\underline{t}_{k}$ lies on the contour $C$, while the notation $(k)=\left(t_{k}, \mathbf{r}_{k}\right)$ is used for space-time variables. With this convention the evolution operator describing the interaction with the external current can be written as

$$
S=T_{C} \exp \left\{-\mathrm{i} \int_{C} \mathrm{~d} \underline{1} \hat{A}_{I}^{\mu}(\underline{1}) J_{\mu}^{(\mathrm{ext})}(\underline{1})\right\},
$$

where $\hat{A}_{I}^{\mu}(\underline{1})=\left(\hat{\phi}_{I}(\underline{1}), \hat{\mathbf{A}}_{I}(\underline{1})\right)$ is the four-potential operator in the interaction picture, and $T_{C}$ is the path-ordering operator on the contour $C$. It is convenient to treat the external charge and current on different branches of the contour $C$ as independent quantities and formally define the ensemble average $O(\underline{1})$ for any Heisenberg operator $\hat{O}(\underline{1})$ as [13]

$$
O(\underline{1})=\left\langle T_{C}\left\{S \hat{O}_{I}(\underline{1})\right\}\right\rangle /\langle S\rangle .
$$

At the end of calculations the physical limit is implied: $\varrho^{(\text {ext })}\left(1_{+}\right)=\varrho^{(\text {ext })}\left(1_{-}\right)$and $\mathbf{J}^{(\text {ext })}\left(1_{+}\right)=$ $\mathbf{J}^{(\mathrm{ext})}\left(1_{-}\right)$. In this limit $\langle S\rangle=1$, and expression (2) goes over into the usual ensemble average of a Heisenberg operator.

The field Green's functions are defined as functional derivatives of the averaged four-potential $A^{\mu}(\underline{1})=\left\langle\hat{A}^{\mu}(\underline{1})\right.$ with respect to the external current:

$$
D^{\mu \nu}(\underline{1} \underline{2})=\delta A^{\mu}(\underline{1}) / \delta J_{\nu}^{(\mathrm{ext})}(\underline{2}) .
$$

In the Coulomb gauge, where $\boldsymbol{\nabla} \cdot \hat{\mathbf{A}}=0$, the components $D^{i j}(\underline{1} \underline{2})$ and $D^{00}(\underline{1} \underline{2})$ describe respectively the transverse (photon) and longitudinal (plasmon) field fluctuations in the plasma. In the physical limit, these Green's functions are given by

$$
\begin{aligned}
& D^{i j}(\underline{1} \underline{2})=-\mathrm{i}\left\langle T_{C} \Delta \hat{A}^{i}(\underline{1}) \Delta \hat{A}^{j}(\underline{2})\right\rangle \\
& D^{00}(\underline{1} \underline{2})=-\mathrm{i}\left\langle T_{C} \Delta \hat{\phi}(\underline{1}) \Delta \hat{\phi}(\underline{2})\right\rangle+\frac{\delta\left(\underline{t}_{1}-\underline{t}_{2}\right)}{4 \pi\left|\mathbf{r}_{1}-\mathbf{r}_{2}\right|}
\end{aligned}
$$


with $\Delta \hat{\mathbf{A}}(\underline{1})=\hat{\mathbf{A}}(\underline{1})-\mathbf{A}(\underline{1})$ and $\Delta \hat{\phi}(\underline{1})=\hat{\phi}(\underline{1})-\phi(\underline{1})$.

To describe field-matter interactions, we also need to introduce the particle Green's functions. Since our main interest is in problems where electrons may be relativistic, we define the electron Green's function in terms of the Dirac field operators:

$$
G(\underline{1} \underline{2})=-\mathrm{i}\left\langle T_{C}\left[S \psi_{I}(\underline{1}) \bar{\psi}_{I}(\underline{2})\right]\right\rangle /\langle S\rangle .
$$

Note that each of the contour components of $G(\underline{1} \underline{2})$ is a $4 \times 4$ spinor matrix. Heavy particles (protons and ions) in real laboratory plasmas are nonrelativistic, so that it is natural to define the corresponding Green's functions as

$$
\mathcal{G}_{B}(\underline{1} \underline{2})=-\mathrm{i}\left\langle T_{C}\left[S \Psi_{B I}(\underline{1}) \Psi_{B I}^{\dagger}(\underline{2})\right]\right\rangle /\langle S\rangle,
$$

where the index " $B$ " labels the particle species, and the operators $\Psi_{B}(\mathbf{r}, t)$ and $\Psi_{B}^{\dagger}(\mathbf{r}, t)$ obey Fermi or Bose commutation rules for equal time arguments.

The first step in developing kinetic theory of multi-component plasmas makes use of Dyson's equations for the field and particle Green's functions $[9,13]$. In the Coulomb gauge, Dyson's equation for the field Green's function (3) reads ${ }^{1}$

$$
-\Delta_{\lambda}^{\mu}(1) D^{\lambda \nu}(\underline{1} \underline{2})=\delta^{\mu \nu}(\underline{1}-\underline{2})+\Pi_{\lambda}^{\mu}\left(\underline{1} \underline{1}^{\prime}\right) D^{\lambda \nu}\left(\underline{1}^{\prime} \underline{2}\right),
$$

where

$$
\Delta_{\nu}^{\mu}(1)=\left(\begin{array}{cccc}
\nabla_{1}^{2} & 0 & 0 & 0 \\
0 & \square_{1} & 0 & 0 \\
0 & 0 & \square_{1} & 0 \\
0 & 0 & 0 & \square_{1}
\end{array}\right),
$$

$\square=\nabla^{2}-\partial^{2} / \partial t^{2}$ is the wave operator, and the matrix $\delta^{\mu \nu}(\underline{1}-\underline{2})$ is given by

$$
\delta^{\mu \nu}(\underline{1}-\underline{2})=\left(\begin{array}{cc}
\delta(\underline{1}-\underline{2}) & 0 \\
0 & -\delta_{i j}^{T}(\underline{1}-\underline{2})
\end{array}\right)
$$

with the transverse delta function satisfying $\nabla_{1 i} \delta_{i j}^{T}(\underline{1}-\underline{2})=0, \nabla_{2 j} \delta_{i j}^{T}(\underline{1}-\underline{2})=0$.

The key quantity describing interactions between the field and the particles is the polarization matrix

$$
\Pi_{\mu \nu}(\underline{1} \underline{2})=\delta J_{\mu}(\underline{1}) / \delta A^{\nu}(\underline{2}) .
$$

The components of the four-vector $J^{\mu}(\underline{1})=\left(\varrho(\underline{1}), \mathbf{J}^{T}(\underline{1})\right)$ are the induced charge density and the induced transverse current density. The matrix $\Pi_{\mu \nu}(\underline{1} \underline{2})$ thus characterizes the system response to variations of the total electromagnetic field.

Dyson's equation for the electron Green's function (6) follows directly from equations of motions for the Dirac field operators $[9,13]$. In the physical limit we have

$$
\left(\mathrm{i} \not \partial_{1}-\mathrm{e} \not A(\underline{1})-m\right) G(\underline{1} \underline{2})-\Sigma\left(\underline{1} \underline{1}^{\prime}\right) G\left(\underline{1}^{\prime} \underline{2}\right)=\delta(\underline{1}-\underline{2})
$$

with the matrix self-energy

$$
\Sigma(\underline{1} \underline{2})=- \text { ie } \gamma_{\mu} G\left(\underline{1}^{\prime}\right) \delta G^{-1}\left(\underline{1}^{\prime} \underline{2}\right) / \delta J_{\mu}^{(\mathrm{ext})}(\underline{1}) .
$$

Finally, nonrelativistic Dyson's equation for the ion Green's function (7) is easily derived by using the equations of motion for the ion field operators $\Psi_{B}(1)$ in the Heisenberg picture. Since in all practical applications the ion transverse current is very small compared to the electron transverse current, we shall neglect the direct interaction between ions and the transverse field $\mathbf{A}$. Within this approximation, one obtains Dyson's equation for the heavy particles (no summation over $B$ )

$$
\left(\mathrm{i} \frac{\partial}{\partial t_{1}}+\frac{\nabla_{1}^{2}}{2 m_{B}}-\mathrm{e}_{B} \phi(\underline{1})\right) \mathcal{G}_{B}(\underline{1} \underline{2})-\Sigma_{B}\left(\underline{1} \underline{1}^{\prime}\right) \mathcal{G}_{B}\left(\underline{1}^{\prime} \underline{2}\right)=\delta(\underline{1}-\underline{2}),
$$

\footnotetext{
${ }^{1}$ Here and in what follows integration over "primed" variables is understood.
} 
where

$$
\Sigma_{B}(\underline{1} \underline{2})=-\mathrm{ie}_{B} \mathcal{G}_{B}\left(\underline{1} \underline{1}^{\prime}\right) \delta \mathcal{G}_{B}^{-1}\left(\underline{1}^{\prime} \underline{2}\right) / \delta \varrho^{(\mathrm{ext})}(\underline{1})
$$

are the ion self-energies.

A convenient way of analysing the polarization matrix and the particle self-energies is to express them in terms of vertex functions. The electron and ion four-vertices are defined as

$$
\Gamma_{\mu}(\underline{1} \underline{2} ; \underline{3})=-\delta G^{-1}(\underline{1} \underline{2}) / \delta A^{\mu}(\underline{3}), \quad \Gamma_{B \mu}(\underline{1} \underline{2} ; \underline{3})=-\delta \mathcal{G}_{B}^{-1}(\underline{1} \underline{2}) / \delta A^{\mu}(\underline{3}) .
$$

Using these definitions, one obtains [9]:

$$
\begin{aligned}
& \Pi_{\mu \nu}(\underline{1} \underline{2})=-\mathrm{i} \operatorname{tr}_{D}\left[\Gamma_{\mu}^{(0)}\left(\underline{1}^{\prime} \underline{2}^{\prime} ; \underline{1}\right) G\left(\underline{2}^{\prime} \underline{3}^{\prime}\right) \Gamma_{\nu}\left(\underline{3}^{\prime} \underline{4}^{\prime} ; \underline{2}\right) G\left(\underline{4}^{\prime} \underline{1}^{\prime}\right)\right] \\
& -\mathrm{i} \sum_{B} \operatorname{tr}_{S}\left[\Gamma_{B \mu}^{(0)}\left(\underline{1}^{\prime} \underline{2}^{\prime} ; \underline{1}\right) \mathcal{G}_{B}\left(\underline{2}^{\prime} \underline{3}^{\prime}\right) \Gamma_{B \nu}\left(\underline{3}^{\prime} \underline{4}^{\prime} ; \underline{2}\right) \mathcal{G}_{B}\left(\underline{4}^{\prime} \underline{1}^{\prime}\right)\right], \\
& \Sigma(\underline{1} \underline{2})=\mathrm{i} \Gamma_{\mu}^{(0)}\left(\underline{1} \underline{1}^{\prime} ; \underline{4}^{\prime}\right) G\left(\underline{1}^{\prime} \underline{2}^{\prime}\right) \Gamma_{\nu}\left(\underline{2}^{\prime} \underline{2} ; \underline{3}^{\prime}\right) D^{\nu \mu}\left(\underline{3}^{\prime} \underline{4}^{\prime}\right), \\
& \Sigma_{B}(\underline{1} \underline{2})=\mathrm{i} \Gamma_{B \mu}^{(0)}\left(\underline{1} \underline{1}^{\prime} ; \underline{4}^{\prime}\right) \mathcal{G}_{B}\left(\underline{1}^{\prime} \underline{2}^{\prime}\right) \Gamma_{B \nu}\left(\underline{2}^{\prime} \underline{2} ; \underline{3}^{\prime}\right) D^{\nu \mu}\left(\underline{3}^{\prime} \underline{4}^{\prime}\right) .
\end{aligned}
$$

The symbols $\operatorname{tr}_{D}$ and $\operatorname{tr}_{S}$ denote the trace over the Dirac spinor indices and the ion spin indices, respectively. The bare four-vertices are given by

$$
\Gamma_{\mu}^{(0)}(\underline{1} \underline{2} ; \underline{3})=\mathrm{e} \delta(\underline{1}-\underline{2}) \delta_{\mu \nu}(\underline{1}-\underline{3}) \gamma^{\nu}, \quad \Gamma_{B \mu}^{(0)}(\underline{1} \underline{2} ; \underline{3})=\mathrm{e}_{B} \delta(\underline{1}-\underline{2}) \delta_{\mu 0}(\underline{1}-\underline{3}) .
$$

Formulas (18) and (19) relate the self-energies to the vertices. There are, however, two other relations between $\Gamma_{\mu}, \Gamma_{B \mu}, \Sigma$, and $\Sigma_{B}$. Using Dyson's equations (12), (14), and recalling (16), it is easy to show that

$$
\Gamma_{\mu}(\underline{1} \underline{2} ; \underline{3})=\Gamma_{\mu}^{(0)}(\underline{1} \underline{2} ; \underline{3})+\delta \Sigma(\underline{1} \underline{2}) / \delta A^{\mu}(\underline{3}), \quad \Gamma_{B \mu}(\underline{1} \underline{2} ; \underline{3})=\Gamma_{B \mu}^{(0)}(\underline{1} \underline{2} ; \underline{3})+\delta \Sigma_{B}(\underline{1} \underline{2}) / \delta A^{\mu}(\underline{3}) .
$$

Equations (8), (12), (14), (17)-(19), and (21), together with Maxwell's equations for the averaged four-potential $A^{\mu}(\mathbf{r}, t)$, provide a basis for studying various transport processes in a manycomponent plasma with relativistic electrons. Although this set of equations is too complicated to solve it in a general form, some self-consistent and tractable approximations can be formulated for weakly coupled plasmas, where collisional interactions are taken into account to lowest orders. As discussed by Bezzerides and DuBois [13], the weak-coupling approximation for a plasma should be regarded as an expansion in terms of the field Green's functions $D_{\mu \nu}$ which are intensity measures for fluctuations of the electromagnetic field, rather than being an asymptotic expansion of vacuum electrodynamics in powers of the fine structure constant $\alpha=\mathrm{e}^{2} / \hbar c$. This scheme applies if several conditions are fulfilled. The first condition reads $\lambda_{\mathrm{pl}} \ll 1$, where $\lambda_{\mathrm{pl}}=1 /\left(n_{e} r_{\mathrm{sc}}^{3}\right)$ is the plasma parameter. Here, $n_{e}$ is the electron number density and $r_{\mathrm{sc}}$ is the screening (Debye) length. The second condition, $\mathrm{e}^{2} /(\hbar v) \ll 1$, where $v$ is a characteristic particle velocity, ensures the validity of the Born approximation for scattering processes. The above conditions are usually fulfilled for relativistic plasmas. Finally, it is assumed that the plasma modes are not excited considerably above their local equilibrium level. This condition is not satisfied for a strongly turbulent regime. In the present work we assume that the system can be described within the weak-coupling approximation.

For a weakly coupled plasma, the vertices can be represented by the Feynman diagrams shown in figure 1. This approximation for the vertices generates the corresponding self-energies and the

$$
\Gamma_{\mu}(\underline{1} \underline{2} ; \underline{3})=\underline{1}_{\underline{2}}^{\mu}+\Gamma_{B \mu}(\underline{1} \underline{2} ; \underline{3})=\underline{1}_{1}^{\mu} \dot{A}^{\mu}+
$$

Figure 1. Lowest order diagrams for the vertices. The first terms are the bare vertices, straight and doubled lines denote respectively $G$ and $\mathcal{G}_{B}$. Dashed lines denote i $D_{\lambda \sigma}$. 


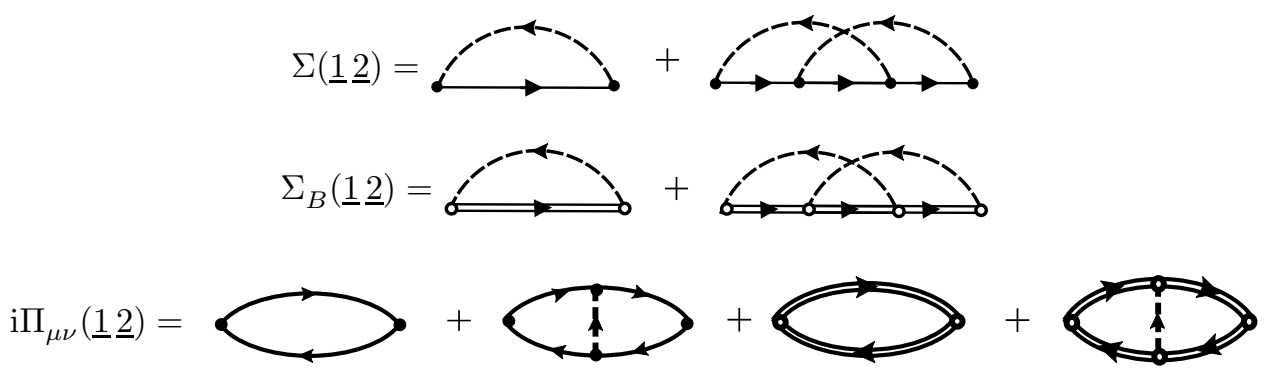

Figure 2. Lowest order diagrams for the electron self-energy (first line), the ion self-energies (second line), and the polarization matrix. The ion contribution to $\Pi_{\mu \nu}$ is obtained as a sum of the last two diagrams over the species index $B$.

polarization matrix shown in figure 2 .

It is, of course, clear that even in the weak-coupling case equations for the field and particle Green's functions are prohibitively difficult to solve, so that one has to introduce further reasonable approximations, depending on the character of the problem. In the present work our interest is with kinetic description of radiative processes in a nonequilibrium relativistic plasma. This description is adequate if the medium is approximately isotropic on the scale of the characteristic wavelengths of field fluctuations and therefore the transverse (photon) and longitudinal (plasmon) modes do not mix. We assume this condition to be satisfied. In this case the field Green's function $D_{\mu \nu}$ and the polarization matrix $\Pi_{\mu \nu}$ can be taken in block form:

$$
D_{\mu \nu}(\underline{1} \underline{2})=\left(\begin{array}{cc}
D(\underline{1} \underline{2}) & 0 \\
0 & D_{i j}(\underline{1} \underline{2})
\end{array}\right), \quad \Pi_{\mu \nu}(\underline{1} \underline{2})=\left(\begin{array}{cc}
\Pi(\underline{1} \underline{2}) & 0 \\
0 & \Pi_{i j}(\underline{1} \underline{2})
\end{array}\right) .
$$

Here $D \equiv D_{00}$ and $\Pi \equiv \Pi_{00}$ are the longitudinal (plasmon) components, whereas $D_{i j}$ and $\Pi_{i j}$ are the transverse (photon) components. Note, however, that the direct coupling between transverse and longitudinal field fluctuations described by the components $D_{0 i}$ and $\Pi_{0 i}$ may be important for long-wavelength and low-frequency modes in plasmas with relativistic electron beams $[14,15]$.

\section{Resonant and virtual photons in plasmas}

Due to the block structure (22) of $D_{\mu \nu}$ and $\Pi_{\mu \nu}$, one obtains from (8) the following equation of motion for the photon Green's functions:

$$
\square_{1} D_{i j}(\underline{1} \underline{2})=\delta_{i j}^{T}(\underline{1}-\underline{2})+\Pi_{i k}\left(\underline{1} \underline{1}^{\prime}\right) D_{k j}\left(\underline{1}^{\prime} \underline{2}\right) .
$$

The adjoint of this equation reads

$$
\square_{2} D_{i j}(\underline{1} \underline{2})=\delta_{i j}^{T}(\underline{1}-\underline{2})+D_{i k}\left(\underline{1} \underline{1}^{\prime}\right) \Pi_{k j}\left(\underline{1}^{\prime} \underline{2}\right) .
$$

A standard way of handling such equations is using the so-called "canonical form" for any function $F(\underline{1} \underline{2})[7]$

$$
F(\underline{1} \underline{2})=\left(\begin{array}{cc}
F\left(1_{+} 2_{+}\right) & F\left(1_{+} 2_{-}\right) \\
F\left(1_{-} 2_{+}\right) & F\left(1_{-} 2_{-}\right)
\end{array}\right)=\left(\begin{array}{cc}
F^{<}(12)+F^{+}(12) & F^{<}(12) \\
F^{>}(12) & F^{<}(12)-F^{-}(12)
\end{array}\right)
$$

with the space-time "correlation functions" $F^{\gtrless}(12)$ and the retarded/advanced functions $F^{ \pm}(12)$ (usually called the "propagators"). In what follows the canonical components of $D_{i j}(\underline{1} \underline{2})$ will be denoted by $d_{i j}^{\gtrless}(12), d_{i j}^{ \pm}(12)$, and the canonical components of $\Pi_{i j}(\underline{1} \underline{2})$ by $\pi_{i j}^{\gtrless}(12), \pi_{i j}^{ \pm}(12)$.

When written in terms of the canonical components, equations (23) and (24) can be transformed into the Kadanoff-Baym equations for the field correlation functions

$$
\begin{aligned}
& \square_{1} d_{i j}^{\gtrless}(12)=\pi_{i k}^{+}\left(11^{\prime}\right) d_{k j}^{\gtrless}\left(1^{\prime} 2\right)+\pi_{i k}^{\gtrless}\left(11^{\prime}\right) d_{k j}^{-}\left(1^{\prime} 2\right), \\
& \square_{2} d_{i j}^{\gtrless}(12)=d_{i k}^{\gtrless}\left(11^{\prime}\right) \pi_{k j}^{-}\left(1^{\prime} 2\right)+d_{i k}^{+}\left(11^{\prime}\right) \pi_{k j}^{\gtrless}\left(1^{\prime} 2\right),
\end{aligned}
$$


and the equations for the photon propagators

$$
\begin{aligned}
& \square_{1} d_{i j}^{ \pm}(12)=\delta_{i j}^{T}(1-2)+\pi_{i k}^{ \pm}\left(11^{\prime}\right) d_{k j}^{ \pm}\left(1^{\prime} 2\right), \\
& \square_{2} d_{i j}^{ \pm}(12)=\delta_{i j}^{T}(1-2)+d_{i k}^{ \pm}\left(11^{\prime}\right) \pi_{k j}^{ \pm}\left(1^{\prime} 2\right)
\end{aligned}
$$

where the primed space-time variables are integrated according to the rule

$$
\int \mathrm{d} 1^{\prime} \ldots=\int_{-\infty}^{\infty} \mathrm{d} t_{1}^{\prime} \int \mathrm{d}^{3} \mathbf{r}_{1}^{\prime} \ldots
$$

Within the framework of kinetic theory, one has to go to the phase description of photon dynamics. This can be achieved by using the Wigner representation for any space-time quantity $F(12) \equiv F\left(x_{1}, x_{2}\right)$ :

$$
F(X, k)=\int \mathrm{d}^{4} x \mathrm{e}^{i k \cdot x} F(X+x / 2, X-x / 2),
$$

where $k \cdot x=k^{\mu} x_{\mu}=k^{0} t-\mathbf{k} \cdot \mathbf{r}$. In order that the kinetic approach may be applicable to electromagnetic fluctuations, it is necessary that the variations of the field Green's functions and the polarization matrix in the space-time variable $X^{\mu}=(T, \mathbf{R})$ are slow on the scales of $\lambda$ and $1 / \omega$, where $\lambda$ and $\omega$ are respectively some characteristic radiation wavelength and frequency. We shall assume these conditions to be satisfied. Then, going over to the Wigner representation in equations for $d_{i j}^{\gtrless}(12)$ and $d_{i j}^{ \pm}(12)$, we keep only terms to the first order in $X$-gradients ${ }^{2}$.

We will not write down the Wigner transformed equations (26) and (27) because it is more convenient to deal with the equivalent transport and the so-called mass-shell equations. The transport equation for $d_{i j}^{\gtrless}(X, k)$ is obtained by taking the difference of equations (26a) and (26b), whereas the mass-shell equation is obtained by taking their sum. In just the same way the transport and mass-shell equations for the propagators $d_{i j}^{ \pm}(X, k)$ can be constructed. By analogy with vacuum QED it is natural to introduce the local principal-axis representation using photon polarization states labelled by $s(s=1,2)$. In this representation we have

$$
d_{i j}^{\gtrless}(X, k)=\sum_{s} \epsilon_{s i}(X, k) d_{s}^{\gtrless}(X, k) \epsilon_{s j}(X, k)
$$

and the analogous expressions for other tensors in terms of the polarization vectors $\boldsymbol{\epsilon}_{s}(X, k)$. Although some care is needed to define properly the local polarization states in an inhomogeneous medium, we will not go here into a detailed discussion of this point (see, e. g., references $[9,13]$ ).

Let us now summarize the resulting first-order gradient equations for the photon modes $s$ in the principal-axis representation. The transport and mass-shell equations for the correlation function read (the arguments $X$ and $k$ are omitted for brevity)

$$
\begin{aligned}
& \left\{k^{2}-\operatorname{Re} \pi_{s}^{+}, d_{s}^{\gtrless}\right\}+\left\{\operatorname{Re} d_{s}^{+}, \pi_{s}^{\gtrless}\right\}=\mathrm{i}\left(\pi_{s}^{>} d_{s}^{<}-\pi_{s}^{<} d_{s}^{>}\right), \\
& \left\{\operatorname{Im} \pi_{s}^{+}, d_{s}^{\gtrless}\right\}+\left\{\operatorname{Im} d_{s}^{+}, \pi_{s}^{\gtrless}\right\}=2\left(k^{2}-\operatorname{Re} \pi_{s}^{+}\right)\left(d_{s}^{\gtrless}-\left|d_{s}^{+}\right|^{2} \pi_{s}^{\gtrless}\right),
\end{aligned}
$$

where

$$
\left\{F_{1}(X, k), F_{2}(X, k)\right\}=\frac{\partial F_{1}}{\partial X^{\mu}} \frac{\partial F_{2}}{\partial k_{\mu}}-\frac{\partial F_{1}}{\partial k^{\mu}} \frac{\partial F_{2}}{\partial X_{\mu}}
$$

is the four-dimensional Poisson bracket. The transport and mass-shell equations for the propagators have a simpler form:

$$
\left\{k^{2}-\pi_{s}^{ \pm}, d_{s}^{ \pm}\right\}=0, \quad\left(k^{2}-\pi_{s}^{ \pm}\right) d_{s}^{ \pm}=1
$$

\footnotetext{
${ }^{2}$ Note that the gradient expansion scheme is a usual way of deriving kinetic equations in the Green's function formalism $[5-7,13]$.
} 
Note that in the last equation the gradient corrections cancel each other, so that one readily finds the "explicit" expression for the propagators

$$
d_{s}^{ \pm}(X, k)=\frac{1}{k^{2}-\operatorname{Re} \pi_{s}^{+}(X, k) \pm \mathrm{i} k_{0} \Gamma_{s}(X, k)}
$$

with the $k$-dependent damping width for the photon mode

$$
\Gamma_{s}(X, k)=-k_{0}^{-1} \operatorname{Im} \pi_{s}^{+}(X, k) .
$$

It is clear that the first of equations (32) is automatically satisfied due to the identity $\{A, f(A)\}=0$.

Equation (30a) may be regarded as a particular case of the gauge-invariant transport equation derived by Bezzerides and DuBois [13] if the polarization states are chosen in accordance with the Coulomb gauge. The mass-shell equation (30b) was ignored in reference [13]. There is no reason, however, to do this because the field correlation functions must satisfy both equations. The massshell equation is in a sense a constraint for approximations in the transport equation.

In the Green's function formalism, transport equations like (30a) are usually regarded as generalized kinetic equations since, in the quasiparticle representation for correlation functions, the left-hand side of a transport equation can be transformed into the drift term, and the right-hand side corresponds to the collision integral. Note, however, that the physical meaning of equation (30a) remains to be seen because the field correlation functions $d_{s}^{\gtrless}(X, k)$ involve contributions from resonant (propagating) and virtual photons. Since a kinetic equation describes only resonant photons, there is a need to pick out the corresponding terms from the field correlation functions. Our analysis of this problem parallels the approach discussed previously by Špička and Lipavský in the context of solid state physics $[16,17]$.

Recalling the definition (31) of the Poisson bracket, it is easy to see that $\left\{k^{2}-\operatorname{Re} \pi_{s}^{+}, d_{s}^{\gtrless}\right\}$ has the structure of the drift term in a kinetic equation for quasiparticles with energies given by the solution of the dispersion equation $k^{2}-\operatorname{Re} \pi_{s}^{+}=0$. But, as was first noted by Botermans and Malfliet [7], terms like the second one on the left-hand side of equation (30a) also contribute to the drift. In the case of photons we have $[9]$

$$
\left\{\operatorname{Re} d_{s}^{+}, \pi_{s}^{\gtrless}\right\}=-\left\{\left(k^{2}-\operatorname{Re} \pi_{s}^{+}\right), \pi_{s}^{\gtrless} \operatorname{Re}\left(d_{s}^{+}\right)^{2}\right\}+\left\{k_{0} \Gamma_{s}, \pi_{s}^{\gtrless} \operatorname{Im}\left(d_{s}^{+}\right)^{2}\right\},
$$

where the first term dominates for weakly damped photon modes. Inserting this expression into equations (30) and introducing new functions $\widetilde{d}_{s}^{\gtrless}(X, k)$ through the relation

$$
d_{s}^{\gtrless}(X, k)=\widetilde{d}_{s}^{\gtrless}(X, k)+\pi_{s}^{\gtrless}(X, k) \operatorname{Re}\left[\left(d_{s}^{+}(X, k)\right)^{2}\right],
$$

we obtain the transport and mass-shell equations for $\widetilde{d}_{s}^{\gtrless}(X, k)$ :

$$
\begin{array}{r}
\left\{k^{2}-\operatorname{Re} \pi_{s}^{+}, \widetilde{d}_{s}^{\gtrless}\right\}+\left\{k_{0} \Gamma_{s}, \pi_{s}^{\gtrless} \operatorname{Im}\left(d_{s}^{+}\right)^{2}\right\}=\mathrm{i}\left(\pi_{s}^{>} \widetilde{d}_{s}^{<}-\pi_{s}^{<} \widetilde{d}_{s}^{>}\right), \\
\left\{k_{0} \Gamma_{s}, \widetilde{d}_{s}^{\gtrless}\right\}+\left\{k^{2}-\operatorname{Re} \pi_{s}^{+}, \pi_{s}^{\gtrless} \operatorname{Im}\left(d_{s}^{+}\right)^{2}\right\}+2\left\{k_{0} \Gamma_{s}, \pi_{s}^{\gtrless} \operatorname{Re}\left(d_{s}^{+}\right)^{2}\right\} \\
=-2\left(k^{2}-\operatorname{Re} \pi_{s}^{+}\right)\left(\widetilde{d}_{s}^{\gtrless}-2\left|d_{s}^{+}\right|^{4}\left(k_{0} \Gamma_{s}\right)^{2} \pi_{s}^{\gtrless}\right) .
\end{array}
$$

It is remarkable that the contributions from the last term of expression (36) to the right-hand side of equation (37a) (collision integral) cancel identically. Physically, this observation suggests that the first term in expression (36) may be regarded as the "resonant" part of the field correlation functions, whereas the second term represents the "off-shell" part which may be identified as the contribution of virtual photons. We would like to emphasize, however, that this interpretation makes sense only in the case of small damping. Formally, the decomposition (36) in itself is not related of course to any interpretation. 
It is instructive to consider the spectral properties of $\widetilde{d}_{s}^{\gtrless}(X, k)$. We first recall that the conventional full spectral function is defined in terms of correlation functions or propagators (see, e. g., reference [7]). For the photon modes, the full spectral function is given by

$$
a_{s}(X, k)=\mathrm{i}\left(d_{s}^{>}-d_{s}^{<}\right)=\mathrm{i}\left(d_{s}^{+}-d_{s}^{-}\right) .
$$

We now introduce the resonant spectral function

$$
\widetilde{a}_{s}(X, k)=\mathrm{i}\left(\tilde{d}_{s}^{>}-\tilde{d}_{s}^{<}\right)
$$

Using formulas (33) and (36), we find that

$$
a_{s}(X, k)=\frac{2 k_{0} \Gamma_{s}}{\left(k^{2}-\operatorname{Re} \pi_{s}^{+}\right)^{2}+\left(k_{0} \Gamma_{s}\right)^{2}}, \quad \widetilde{a}_{s}(X, k)=\frac{4\left(k_{0} \Gamma_{s}\right)^{3}}{\left[\left(k^{2}-\operatorname{Re} \pi_{s}^{+}\right)^{2}+\left(k_{0} \Gamma_{s}\right)^{2}\right]^{2}} .
$$

Here two important observations can be made. First, it is easy to check that both spectral functions take the same form in the zero damping limit:

$$
\lim _{\Gamma_{s} \rightarrow 0} a_{s}=\lim _{\Gamma_{s} \rightarrow 0} \widetilde{a}_{s}=2 \pi \eta\left(k_{0}\right) \delta\left(k^{2}-\operatorname{Re} \pi_{s}^{+}\right),
$$

where $\eta\left(k_{0}\right)=k_{0} /\left|k_{0}\right|$. Second, for a finite $\Gamma_{s}$, the resonant spectral function falls off faster than the full spectral function. In other words, $\widetilde{a}_{s}$ has a stronger peak and smaller wings than $a_{s}$. Thus, for weakly damped modes, the first term in formula (36) dominates in the vicinity of the photon mass-shell $\left(k^{2} \approx \operatorname{Re} \pi_{s}^{+}\right)$, while the second term dominates in the off-shell region where it falls as $\left(k^{2}-\operatorname{Re} \pi_{s}^{+}\right)^{-2}$. This supports the above interpretation of decomposition (36).

Expression (40) for $\widetilde{a}_{s}$ may look not too familiar since quasiparticle spectral functions are usually taken in the Lorentzian form like $a_{s}$. Note, however, that the spectral function $\widetilde{a}_{s}$ also occurs in the self-consistent calculation of thermodynamic quantities of equilibrium QED plasmas. As shown by Vanderheyden and Baym [18], the resonant spectral function rather than the Lorentzian-like full spectral function determines the contribution of photons to the equilibrium entropy. That is why in reference [18] $\widetilde{a}_{s}$ was called "the entropy spectral function". We have seen, however, that this function has a deeper physical sense; it characterizes the spectral properties of resonant (propagating) photons in a medium. Therefore, we prefer to call $\widetilde{a}_{s}$ the resonant spectral function.

\section{Kinetic equation for resonant photons}

In order to convert equation (37a) into the photon kinetic equation, we introduce the following representation for the resonant parts of the field correlation functions:

$$
\widetilde{d}_{s}^{<}(X, k)=-\mathrm{i} \widetilde{a}_{s}(X, k) N_{s}^{<}(X, k), \quad \widetilde{d}_{s}^{>}(X, k)=-\mathrm{i} \widetilde{a}_{s}(X, k) N_{s}^{>}(X, k),
$$

where new functions $N_{s}^{\gtrless}(X, k)$ satisfy $N_{s}^{>}(X, k)-N_{s}^{<}(X, k)=1$. It is also convenient to use the representation

$$
\begin{aligned}
& N_{s}^{<}(X, k)=\theta\left(k_{0}\right) N_{s}(X, k)-\theta\left(-k_{0}\right)\left[1+N_{s}(X,-k)\right], \\
& N_{s}^{>}(X, k)=\theta\left(k_{0}\left[1+N_{s}(X, k)\right]-\theta\left(-k_{0}\right) N_{s}(X,-k),\right.
\end{aligned}
$$

which serves as the definition of the local photon distribution function $N_{s}(X, k)$ in four-dimensional phase space. In the above relations $\theta(x)$ is the conventional step function, i. e., $\theta(x)=0$ for $x<0$, and $\theta(x)=1$ for $x>0$.

For our purposes, it suffices to consider only equation (37a) for $\widetilde{d}_{s}^{<}(X, k)$ since the transport equation for $\widetilde{d}_{s}^{>}(X, k)$ leads to the same kinetic equation. Using representation (42) and expression (40) for the resonant spectral function, some algebra detailed in reference [9] gives

$$
\widetilde{a}_{s}\left[\left\{k^{2}-\operatorname{Re} \pi_{s}^{+}, N_{s}^{<}\right\}-\frac{k^{2}-\operatorname{Re} \pi_{s}^{+}}{k_{0} \Gamma_{s}}\left\{k_{0} \Gamma_{s}, N_{s}^{<}\right\}-\mathrm{i}\left(\pi_{s}^{>} N_{s}^{<}-\pi_{s}^{<} N_{s}^{>}\right)\right]=0 .
$$


The desired photon kinetic equation is obtained by setting the expression in square brackets equal to zero. Applying the same procedure to the mass-shell equation (37b) leads to

$$
\left(k^{2}-\operatorname{Re} \pi_{s}^{+}\right) \widetilde{a}_{s}[\ldots]=0
$$

with the same expression in square brackets as in equation (44). We see that in the present approach the mass-shell equation is consistent with the kinetic equation.

We have seen that $\widetilde{a}_{s}(X, k)$ is a sharply peaked function of $k_{0}$ near the effective photon frequencies, $\omega_{s}(X, \mathbf{k})$, which are solutions of the dispersion equation $k^{2}-\operatorname{Re} \pi_{s}^{+}(X, k)=0$. For definiteness, we assume that the photon frequencies are positive solutions. If $k_{0}=\omega_{s}(X, \mathbf{k})$ is such a solution, then, using the property $\left[\pi_{s}^{+}(k)\right]^{*}=\pi_{s}^{+}(-k)$, it is easy to check that the corresponding negative solution is $k_{0}=-\omega_{s}(X,-\mathbf{k})$. In the small damping limit, the resonant spectral function may be approximated as (see equation (41))

$$
\widetilde{a}_{s}(X, k)=2 \pi \eta\left(k_{0}\right) \delta\left(k^{2}-\operatorname{Re} \pi_{s}^{+}\right) .
$$

Then, integrating equation (44) over $k_{0}>0$, we arrive at the kinetic equation

$$
\begin{gathered}
\left(\frac{\partial}{\partial T}+\frac{\partial \omega_{s}(X, \mathbf{k})}{\partial \mathbf{k}} \cdot \frac{\partial}{\partial \mathbf{R}}-\frac{\partial \omega_{s}(X, \mathbf{k})}{\partial \mathbf{R}} \cdot \frac{\partial}{\partial \mathbf{k}}\right) n_{s}(X, \mathbf{k})=\mathrm{i} Z_{s}(X, \mathbf{k}) \pi_{s}^{<}(X, \mathbf{k})\left[1+n_{s}(X, \mathbf{k})\right] \\
-\mathrm{i} Z_{s}(X, \mathbf{k}) \pi_{s}^{>}(X, \mathbf{k}) n_{s}(X, \mathbf{k}),
\end{gathered}
$$

where

$$
n_{s}(X, \mathbf{k})=\left.N_{s}(X, k)\right|_{k_{0}=\omega_{s}(X, \mathbf{k})}
$$

is the on-shell photon distribution function, $\pi_{s}^{\gtrless}(X, \mathbf{k})=\left.\pi_{s}^{\gtrless}(X, k)\right|_{k_{0}=\omega_{s}(X, \mathbf{k})}$, and the "wavefunction renormalization" $Z_{s}(X, \mathbf{k})$ is given by

$$
Z_{s}^{-1}(X, \mathbf{k})=\left.\frac{\partial}{\partial k_{0}}\left(k^{2}-\operatorname{Re} \pi_{s}^{+}(X, k)\right)\right|_{k_{0}=\omega_{s}(X, \mathbf{k})} .
$$

The photon kinetic equation (47) was derived many years ago by DuBois [19] for nonrelativistic plasmas. The generalization to relativistic plasmas is obvious until the polarization functions $\pi_{s}^{\gtrless}(X, k)$ are specified. There was a reason, however, to discuss here the derivation of the photon kinetic equation. As we have seen, the kinetic equation is only related to the resonant parts of the transverse field correlation functions while their off-shell parts are represented by the last term in formula (36). The photon distribution function is usually introduced through the relations $d_{s}^{\gtrless}=-\mathrm{i} a_{s} N_{s}^{\gtrless}$ with the full spectral function $a_{s}$ which is then taken in the singular form (46) (see, e. g., reference [19]). In doing so, the off-shell corrections to $d_{s}^{\gtrless}$ are missing. As we shall see below, these corrections contribute to the transverse polarization functions $\pi_{s}^{\gtrless}$ determining the photon emission and absorption rates.

The first and the second terms on the right-hand side of equation (47) are respectively the photon emission and absorption rates. We recall that these kinetic results imply the singular approximation (46) for the resonant spectral function. It is possible, however, to derive a general expression for the average electromagnetic energy production, in which the finite width of $\widetilde{a}_{s}(X, k)$ is taken into account [9]. The quantity of special interest is the differential radiating power associated with the photon production:

$$
\frac{\mathrm{d} R(X, k)}{\mathrm{d}^{4} k}=\frac{\mathrm{i}}{(2 \pi)^{4}} k_{0} \sum_{s} \widetilde{a}_{s}(X, k) \pi_{s}^{<}(X, k)\left[1+N_{s}(X, k)\right],
$$

where $k_{0}>0$ is implied. It is seen that, for a fixed $\mathbf{k}$, the $\widetilde{a}_{s}(X, k)$ is just the weight function that determines the emission profile. This supports once again the interpretation of $\widetilde{a}_{s}(X, k)$ as the 
spectral function for propagating photons in a plasma. Within approximation (46), the differential radiating power can be expressed in terms of the three-momentum $\mathbf{k}$ :

$$
\frac{\mathrm{d} R(X, \mathbf{k})}{\mathrm{d}^{3} \mathbf{k}}=\frac{\mathrm{i}}{(2 \pi)^{3}} \sum_{s} \omega_{s}(X, \mathbf{k}) Z_{s}(X, \mathbf{k}) \pi_{s}^{<}(X, \mathbf{k})\left[1+n_{s}(X, \mathbf{k})\right] .
$$

As one would expect, this expression is consistent with the photon production rate in the kinetic equation (47).

\section{Transverse polarization functions}

The transverse polarization functions $\pi_{s}^{\gtrless}(X, k)$ are key quantities in computing radiation effects. Here we shall restrict our discussion to the polarization function $\pi_{s}^{<}(X, k)$ that determines the local radiating power. The starting point for the calculation of this function is the polarization matrix $\Pi_{\mu \nu}(\underline{1} \underline{2})$ represented by the diagrams shown in figure 2 . Recalling the definition (20) of the bare ion

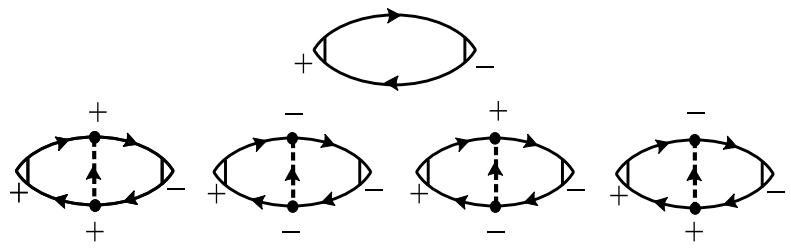

Figure 3. Space-time diagrams for $\mathrm{i} \pi_{i j}^{<}(12)$. The \pm signs indicate the branches of the contour $C$.

vertex, it is easy to verify that the last two diagrams do not contribute to $\Pi_{i j}(\underline{1} \underline{2})$. Its component $\pi_{i j}^{<}(12)=\Pi_{i j}\left(1_{+} 2_{-}\right)$is thus given by the diagrams shown in figure 3 , where we have introduced the transverse bare vertex

$$
\Gamma_{i}(12 ; 3)={ }_{1}^{i, 3} \equiv-\mathrm{e} \delta(1-2) \delta_{i j}^{T}(1-3) \gamma^{j}
$$

The next step is to compute the diagrams in figure 3 by Wigner transforming all functions and then using the principal-axis representation $(29)$ for $\pi_{i j}^{<}(X, k)$. Since the polarization functions will be used for the calculation of the local radiating power, the $X$-gradient corrections to Green's functions can be omitted. Then the contribution of the first (one-loop) diagram in figure 3 to $\pi_{s}^{<}(X, k)$ is

$$
\pi_{s}^{<(1)}(X, k)=-\mathrm{ie}^{2} \int \frac{\mathrm{d}^{4} p}{(2 \pi)^{4}} \operatorname{tr}_{D}\left\{\phi_{s}(k) G^{<}(X, p) \oiint_{s}(k) G^{>}(X, p-k)\right\}
$$

with the polarization four-vectors $\epsilon_{s}^{\mu}(X, k)=\left(0, \epsilon_{s}(X, k)\right)$.

The computation of the diagrams in the second line of figure 3 requires a more elaborate analysis. Some of these diagrams give small corrections to the one-loop expression (53) and therefore may be omitted. For a detailed discussion of this point we refer to the paper [9]. The contribution of relevant diagrams describing higher-order radiative processes (e. g., bremsstrahlung and Compton scattering) is given by (the fixed argument $X$ is omitted)

$$
\begin{gathered}
\pi_{s}^{<(2)}(k)=\frac{\mathrm{e}^{4}}{(2 \pi)^{8}} \int \mathrm{d}^{4} k^{\prime} \prod_{i=1}^{4} \mathrm{~d}^{4} p_{i} \delta^{4}\left(k-p_{2}+p_{3}\right) \delta^{4}\left(k^{\prime}-p_{3}+p_{4}\right) \delta^{4}\left(p_{1}+p_{3}-p_{2}-p_{4}\right) \\
\times\left[D_{\lambda \lambda^{\prime}}^{<}\left(k^{\prime}\right) \operatorname{tr}_{D}\left\{\phi_{s}(k) G^{<}\left(p_{1}\right) \hat{\gamma}^{\lambda^{\prime}}\left(k^{\prime}\right) G^{-}\left(p_{2}\right) \phi_{s}(k) G^{>}\left(p_{3}\right) \hat{\gamma}^{\lambda}\left(k^{\prime}\right) G^{-}\left(p_{4}\right)\right\}\right. \\
\left.\quad+D_{\lambda \lambda^{\prime}}^{>}\left(k^{\prime}\right) \operatorname{tr}_{D}\left\{\phi_{s}(k) G^{+}\left(p_{1}\right) \hat{\gamma}^{\lambda^{\prime}}\left(k^{\prime}\right) G^{<}\left(p_{2}\right) \phi_{s}(k) G^{+}\left(p_{3}\right) \hat{\gamma}^{\lambda}\left(k^{\prime}\right) G^{>}\left(p_{4}\right)\right\}\right] .
\end{gathered}
$$

We have introduced the notation $\hat{\gamma}^{\mu}(k)=\left(\gamma^{0}, \Delta_{i j}^{\perp}(\mathbf{k}) \gamma^{j}\right)$, where $\Delta_{i j}^{\perp}(\mathbf{k})=\delta_{i j}-k_{i} k_{j} /|\mathbf{k}|^{2}$ is the transverse projector. Expressions (53) and (54) provide a basis for computing the local radiating power in weakly coupled relativistic plasmas. 


\section{Extended quasiparticle approximation for relativistic electrons}

It is clear that explicit calculations of $\pi_{s}^{<}(X, k)$ require a knowledge of the electron propagators $G^{ \pm}(X, p)$ and correlation functions $G^{\gtrless}(X, p)$. Equations for the corresponding space-time quantities $G^{ \pm}(12)$ and $G^{\gtrless}(12)$ can be derived from Dyson's equation (12) using the canonical form (25) of the electron Green's function $G(\underline{1} \underline{2})$ and the self-energy $\Sigma(\underline{1} \underline{2})$. The derivation follows a standard way (see, e. g., reference [7]), so that we will not discuss it here.

Due to the spinor structure of the Wigner transformed propagators $G^{ \pm}(X, p)$, they contain first-order $X$-gradient corrections [9] but, in calculating the local radiating power, one can use the local propagators

$$
g^{ \pm}(X, p)=\frac{1}{\not I(X, p)-m-\Sigma^{ \pm}(X, p)},
$$

where $\Pi^{\mu}(X, p)=p^{\mu}-e A^{\mu}(X)$ is the kinematic momentum. Note that the appearance of the vector potential in the above expression and other formulas is due to the fact that the electron Green's function (6) is not invariant under gauge transformation of the mean electromagnetic field. One must be careful to distinguish between the kinematic $(\Pi)$ and the canonical $(p)$ momenta in evaluating the drift terms in a kinetic equation for electrons, because the Poisson brackets contain derivatives of $A(X)$. On the other hand, for local quantities, such as the emission and absorption rates, the vector potential $A(X)$ drops out from the final expressions as it must be due to gauge invariance. Formally, this can be achieved by the change of variables $p_{i} \rightarrow p_{i}+e A(X)$ in integrals over the electron four-momenta, or equivalently by setting $A(X)=0[9]$. From now on we use the latter prescription.

As for the correlation functions $G^{\gtrless}(X, p)$, the main problem is to express them in terms of the electron (positron) distribution functions. A simple quasiparticle ansatz for relativistic electrons was proposed by Bezzerides and DuBois [13] (see also below) but it cannot be used to calculate the polarization functions $\pi_{s}^{\gtrless}(X, k)$. The point is that the electron correlation functions $G^{\gtrless}(X, p)$ contain not only sharply peaked "quasiparticle" parts but also off-shell parts which contribute to the emission and absorption rates. The situation is strongly reminiscent of our previous discussion of the field correlation functions, so that it is natural to follow the same way by analyzing drift terms in transport equations derived from the Kadanoff-Baym equations for $G^{\gtrless}(X, p)$. This is detailed in references $[9,20]$. Here we quote the resulting decomposition of the electron correlation functions in the local approximation (cf. formula (36) for photons):

$$
G^{\gtrless}(X, p)=\widetilde{G}^{\gtrless}(X, p)+\frac{1}{2}\left[g^{+}(X, p) \Sigma^{\gtrless}(X, p) g^{+}(X, p)+g^{-}(X, p) \Sigma^{\gtrless}(X, p) g^{-}(X, p)\right] .
$$

To show that the $\widetilde{G}^{\gtrless}(X, p)$ may be interpreted as the quasiparticle parts of the electron correlation functions, it is instructive to consider their spectral properties. The full spectral function in spinor space is defined as $[7,21]$

$$
\mathcal{A}(X, p)=\mathrm{i}\left(G^{>}(X, p)-G^{<}(X, p)\right)=\mathrm{i}\left(G^{+}(X, p)-G^{-}(X, p)\right) .
$$

In the local approximation $G^{ \pm}(X, p)=g^{ \pm}(X, p)$. We also introduce the quasiparticle spectral function associated with $\widetilde{G}^{\gtrless}(X, p)$ :

$$
\widetilde{\mathcal{A}}(X, p)=\mathrm{i}\left(\widetilde{G}^{>}(X, p)-\widetilde{G}^{<}(X, p)\right) .
$$

Then, recalling formulas $(55),(56)$, and the relation $\Sigma^{>}-\Sigma^{<}=\Sigma^{+}-\Sigma^{-}$, we find that

$$
\mathcal{A}(X, p)=\mathrm{i} g^{+} \Delta \Sigma g^{-}, \quad \widetilde{\mathcal{A}}(X, p)=-\frac{\mathrm{i}}{2} g^{+} \Delta \Sigma g^{+} \Delta \Sigma g^{-} \Delta \Sigma g^{-},
$$

where $\Delta \Sigma(X, p)=\Sigma^{+}(X, p)-\Sigma^{-}(X, p)$. To gain some insight into an important difference between the spectral functions $\mathcal{A}(X, p)$ and $\widetilde{\mathcal{A}}(X, p)$, let us use a simple ansatz [22-24]

$$
\Sigma^{ \pm}(X, p)=\mp \mathrm{i}\left(\Gamma_{p} / 2\right) \gamma^{0}
$$


where $\Gamma_{p}>0$ is a "spectral width parameter". Then, assuming $\Gamma_{p} \ll E_{p}$, where $E_{p}=\sqrt{\mathbf{p}^{2}+m^{2}}$, some spinor algebra leads to [9]

$$
\mathcal{A}(X, p) \approx \frac{2 p_{0} \Gamma_{p}}{\left(p_{0}^{2}-E_{p}^{2}\right)^{2}+\left(p_{0} \Gamma_{p}\right)^{2}}(\not p+m), \quad \widetilde{\mathcal{A}}(X, p) \approx \frac{4\left(p_{0} \Gamma_{p}\right)^{3}}{\left[\left(p_{0}^{2}-E_{p}^{2}\right)^{2}+\left(p_{0} \Gamma_{p}\right)^{2}\right]^{2}}(\not p+m) .
$$

In the limit $\Gamma_{p} \rightarrow 0$ both spectral functions take the same form

$$
\lim _{\Gamma_{p} \rightarrow 0} \widetilde{\mathcal{A}}=\lim _{\Gamma_{p} \rightarrow 0} \mathcal{A}=2 \pi \eta\left(p_{0}\right) \delta\left(p^{2}-m^{2}\right)(\not p+m)
$$

but, for a finite $\Gamma_{p}$, the quasiparticle spectral function falls off faster than the full spectral function. In other words, the "collisional broadening" of $\widetilde{\mathcal{A}}(X, p)$ function is considerably smaller than that of $\mathcal{A}(X, p)$. This has much in common with the properties of the resonant and full spectral functions for transverse field fluctuations (cf. expressions (40)).

In analogy with the kinetic description of resonant photons in section 4 , it is reasonable to introduce the electron (positron) distribution functions through relations between $\widetilde{G}^{\gtrless}(X, p)$ and the quasiparticle spectral function. As shown in reference [9], for small quasiparticle damping and equal probabilities of the spin polarization these relations can be taken in the form

$$
\widetilde{G}^{\gtrless}(X, p)=\mp \mathrm{i} \widetilde{\mathcal{A}}(X, p) f^{\gtrless}(X, p),
$$

where the $f^{\gtrless}(X, p)$ are expressed in terms of the distribution functions for electrons $\left(f_{\mathrm{e}^{-}}\right)$and positrons $\left(f_{\mathrm{e}^{+}}\right)$:

$$
\begin{array}{ll}
\left.f^{<}(X, p)\right|_{p_{0}>0}=f_{\mathrm{e}^{-}}(X, \mathbf{p}), & \left.f^{<}(X, p)\right|_{p_{0}<0}=1-f_{\mathrm{e}^{+}}(X,-\mathbf{p}), \\
\left.f^{>}(X, p)\right|_{p_{0}>0}=1-f_{\mathrm{e}^{-}}(X, \mathbf{p}), & \left.f^{>}(X, p)\right|_{p_{0}<0}=f_{\mathrm{e}^{+}}(X,-\mathbf{p}) .
\end{array}
$$

Neglecting the second (off-shell) term in formula (56), i. e., identifying $\widetilde{G}^{\gtrless}(X, p)$ with the full correlation functions, and using the singular form (62) for $\widetilde{\mathcal{A}}(X, p)$ in relation (63), we recover the relativistic quasiparticle approximation used by Bezzerides and DuBois [13]. This approximation is sufficient to derive a particle kinetic equation in which the collision term involves electron-electron (electron-positron) scattering but, as shown below, is inadequate to describe radiative processes (e. g., Compton scattering and bremsstrahlung). Therefore, the off-shell parts of $G^{\gtrless}(X, p)$ are to be taken into account.

To the first order in the field fluctuations, we have [9]

$$
\Sigma^{\gtrless}(X, p)=\mathrm{ie}^{2} \int \frac{\mathrm{d}^{4} k}{(2 \pi)^{4}} \hat{\gamma}^{\mu}(k) G^{\gtrless}(X, p+k) \hat{\gamma}^{\nu}(k) D_{\nu \mu}^{\lessgtr}(X, k) .
$$

We see that formula (56) is in fact an integral equation for $G^{\gtrless}(X, p)$. Retaining only terms linear in $D_{\mu \nu}^{\lessgtr}(X, k)$ yields

$$
\begin{aligned}
G^{\gtrless}(X, p)=\widetilde{G}^{\gtrless}(X, p)+\mathrm{i} \frac{\mathrm{e}^{2}}{2} \int \frac{\mathrm{d}^{4} k}{(2 \pi)^{4}} D_{\nu \mu}^{\lessgtr}(k)\left[g^{+}(p) \hat{\gamma}^{\mu}(k) \widetilde{G}^{\gtrless}(p+k) \hat{\gamma}^{\nu}(k) g^{+}(p)\right. \\
\left.+g^{-}(p) \hat{\gamma}^{\mu}(k) \widetilde{G}^{\gtrless}(p+k) \hat{\gamma}^{\nu}(k) g^{-}(p)\right] .
\end{aligned}
$$

In non-relativistic kinetic theory, an analogous decomposition of correlation functions into the quasiparticle and off-shell parts is called the "extended quasiparticle approximation" [16,17,25]. Expression (66) may thus be regarded as the extended quasiparticle approximation for relativistic electrons. 


\section{Photon production: elementary processes}

We can now express the relevant contributions to $\pi_{s}^{<}(X, k)$ in terms of the quasiparticle parts of the electron correlation functions. Since our consideration is restricted to the lowest order in the field fluctuations, the full electron correlation functions $G^{\gtrless}(X, p)$ entering expression (54) are to be replaced by $\widetilde{G}^{\gtrless}(X, p)$. Note, however, that in formula (53) one must keep first-order off-shell corrections to the electron correlation functions coming from the integral term in equation (66). These corrections are to be combined with the term (54). Collecting all contributions to $\pi_{s}^{<}(X, k)$, it is convenient to eliminate the field correlation functions $D_{\mu \nu}^{>}(X, k)$ with the help of the symmetry relation $D_{\mu \nu}^{>}(X, k)=D_{\nu \mu}^{<}(X,-k)$. Then some algebra gives

$$
\begin{array}{r}
\pi_{s}^{<}(X, k)=-\mathrm{ie}^{2}(2 \pi)^{4} \int \frac{\mathrm{d}^{4} p_{1}}{(2 \pi)^{4}} \frac{\mathrm{d}^{4} p_{2}}{(2 \pi)^{4}} \delta^{4}\left(p_{1}-p_{2}-k\right) \operatorname{tr}_{D}\left\{\phi_{s}(k) \widetilde{G}^{<}\left(p_{1}\right) \phi_{s}(k) \widetilde{G}^{>}\left(p_{2}\right)\right\} \\
+\mathrm{e}^{4}(2 \pi)^{4} \int \frac{\mathrm{d}^{4} p_{1}}{(2 \pi)^{4}} \frac{\mathrm{d}^{4} p_{2}}{(2 \pi)^{4}} \frac{\mathrm{d}^{4} k^{\prime}}{(2 \pi)^{4}} \delta^{4}\left(p_{1}+k^{\prime}-p_{2}-k\right) K_{s}^{\lambda \lambda^{\prime}}\left(p_{1}, p_{2} ; k, k^{\prime}\right) D_{\lambda \lambda^{\prime}}^{<}\left(k^{\prime}\right) .
\end{array}
$$

The explicit expression for $K_{s}^{\lambda \lambda^{\prime}}\left(p_{1}, p_{2} ; k, k^{\prime}\right)$ is rather cumbersome and therefore is not given here (see reference [9]). We only note that it contains the Dirac traces of different terms involving the electron propagators $g^{ \pm}$and the quasiparticle correlation functions $\widetilde{G}^{\gtrless}$.

Let us discuss shortly some radiative processes described by the polarization function (67). We begin with the contribution of the first term to the radiating power (51). Usually the corresponding process is referred to as Cherenkov radiation. Note, however, that the energy-momentum conserving Cherenkov emission of photons in plasmas is kinematically forbidden [13] because, for all the expected densities, the dispersion curve for transverse ways stays above the $\omega=|\mathbf{k}|$. Strictly speaking, this statement is only true when the quasiparticle spectral function $\widetilde{\mathcal{A}}(p)$ has the singular mass-shell form (62), i. e., all medium effects are ignored. The collisional broadening of the $\widetilde{\mathcal{A}}(p)$ results in a statistical energy uncertainty for electrons (positrons), and the emission of low-energy photons becomes possible ${ }^{3}$. Neglecting the positron contribution to the radiating power and assuming, for simplicity, equal probabilities for the photon polarizations and the particle spin states, one obtains [9] (the fixed argument $X$ is omitted)

$$
\left(\frac{\mathrm{d} R(\mathbf{k})}{\mathrm{d}^{3} \mathbf{k}}\right)_{\text {Cherenkov }}=\frac{\mathrm{e}^{2}}{(2 \pi)^{3}}[1+n(\mathbf{k})] \int \frac{\mathrm{d}^{3} \mathbf{p}}{(2 \pi)^{3}} \Lambda(\mathbf{p}, \mathbf{k}) f(\mathbf{p}),
$$

where $n(\mathbf{k}) \equiv n_{s}(\mathbf{k})$ and $f(\mathbf{p})$ is the electron distribution function. With the parametrization (61) of the quasiparticle spectral function, the transition probability $\Lambda(\mathbf{p}, \mathbf{k})$ can be calculated analytically:

$$
\Lambda(\mathbf{p}, \mathbf{k})=\frac{\Gamma_{p}^{3}\left(\mathbf{p}^{2}-(\mathbf{p} \cdot \mathbf{k})^{2} / \mathbf{k}^{2}\right)\left\{5 \Gamma_{p}^{2}+\omega^{2}(\mathbf{k})\left[1-(\mathbf{p} \cdot \mathbf{k}) /\left(E_{p} \omega(\mathbf{k})\right)\right]^{2}\right\}}{E_{p}^{2}\left\{\Gamma_{p}^{2}+\omega^{2}(\mathbf{k})\left[1-(\mathbf{p} \cdot \mathbf{k}) /\left(E_{p} \omega(\mathbf{k})\right)\right]^{2}\right\}^{3}} .
$$

Since in a plasma $\left[1-(\mathbf{p} \cdot \mathbf{k}) /\left(E_{p} \omega(\mathbf{k})\right)\right] \neq 0$ for all possible $\mathbf{p}$ and $\mathbf{k}$, it is evident that $\Lambda(\mathbf{p}, \mathbf{k}) \rightarrow 0$ in the limit $\Gamma_{p} \rightarrow 0$ (more precisely, $\Gamma_{p} / \omega(\mathbf{k}) \rightarrow 0$ ). Finally, we note that for fixed $\mathbf{p}$ and $\omega(\mathbf{k})$, the quantity $\Lambda(\mathbf{p}, \mathbf{k}) \Gamma_{p}$ may be regarded as the angular distribution of emitted photons. The behavior of this distribution in the frequency region $\omega(\mathbf{k}) \approx \Gamma_{p}$ is illustrated in figure 4 .

To understand the radiative processes associated with the second term in expression (67), we note that, according to (22), the field correlation functions are represented by the block matrix

$$
D_{\mu \nu}^{\gtrless}(X, k)=\left(\begin{array}{cc}
D^{\gtrless}(X, k) & 0 \\
0 & \sum_{s} \epsilon_{s i}(k) d_{s}^{\gtrless}(X, k) \epsilon_{s j}(k)
\end{array}\right) .
$$

\footnotetext{
${ }^{3} \mathrm{~A}$ similar situation occurs in the case of a quark-gluon plasma [22] where the thermal broadening of the quark spectral function is one of the mechanisms for the soft photon production.
} 

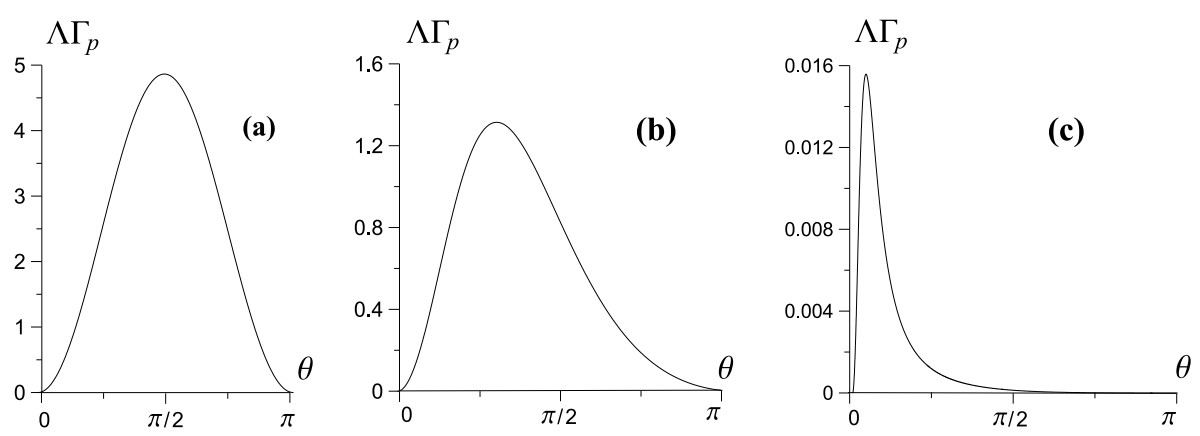

Figure 4. Angular distribution of emitted photons in the Cherenkov channel: (a) $\omega(\mathbf{k})=0.1 \Gamma_{p}$; (b) $\omega(\mathbf{k})=\Gamma_{p} ;(\mathbf{c}) \omega(\mathbf{k})=10 \Gamma_{p}$. Here, $\theta$ is the angle between the electron momentum $\mathbf{p}$ and the photon momentum $\mathbf{k}$. The electron kinetic energy is assumed to be $E_{\text {kin }}=10 \mathrm{MeV}$.

We, therefore, have two different contributions to the radiating power arising from the interaction of electrons (positrons) with longitudinal and transverse fluctuations of the electromagnetic field. The contribution to the radiating power coming from the resonant term in decomposition (36) corresponds to Compton scattering and electron-positron annihilation in the plasma [9]. It is important to emphasize that the cross section for these processes (the Klein-Nishina formula) known from vacuum QED [26] is recovered if the collisional broadening of the electron propagators $g^{ \pm}(p)$ and the quasiparticle spectral function $\widetilde{\mathcal{A}}(p)$ is neglected. There is another contribution to the radiating power associated with the second (off-shell) part of the field correlation function $d_{s}^{<}(k)$ given by equation (36). Since the transverse polarization effects are caused by current fluctuations, this contribution may be interpreted physically as coming from the scattering of electrons by current fluctuations in the plasma. Finally, the contribution to the radiating power coming from the longitudinal field correlation function $D^{<}(X, k)$ corresponds to the scattering of electrons by ions (bremsstrahlung), one-photon electron-positron annihilation, and the scattering of electrons by charge fluctuations (plasmons). The vacuum QED cross section for bremsstrahlung emission of photons (the Bethe-Heitler formula) [26] is recovered if the medium effects are neglected. For a detailed discussion of all these points we refer to the work [9].

\section{Summary}

One of the most important features of photon kinetics is the crucial role played by the off-shell parts of the particle and field correlation functions in the derivation of the photon emission rate. Note that in vacuum QED the separation of on-shell and off-shell states is in fact trivial, since the on-shell states correspond to incoming or outgoing particles in a scattering process while the off-shell (virtual) states occur in the calculation of the S-matrix. In kinetic theory, however, one is dealing with ensemble averaged correlation functions involving both the resonant (quasiparticle) and off-shell parts. We have seen that, for weakly coupled plasmas, the structure of drift terms in the gradient-expanded transport equations provides a useful guide to separate the quasiparticle and off-shell contributions to the correlation functions. Note that off-shell transport is also a problem of great interest for a proper dynamical treatment of stable particles and broad resonances in a dense nuclear medium [27], but in that case it is hard to separate unambiguously the quasiparticle and off-shell contributions to correlation functions because of strong medium effects.

\section{Acknowledgement}

This work was partially supported by the German Research Society (DFG) under Grant SFB 652. 


\section{References}

1. Bogolyubov N.N. Problems of Dynamic Theory in Statistical Physics. Gostekhizdat, MoscowLeningrad, 1946 (in Russiain) [Reprinted in: Studies in Statistical Mechanics (de Boer J. and Uhlenbeck G.E., eds.). Vol. 1, North-Holland, Amsterdam, 1962].

2. Zubarev D.N., Morozov V.G., Röpke G. Statistical Mechanics of Nonequilibrium Processes, Vol. 1. Akademie Verlag, Berlin, 1996.

3. Zubarev D.N., Morozov V.G., Röpke G. Statistical Mechanics of Nonequilibrium Processes, Vol. 2. Akademie Verlag, Berlin, 1997.

4. Bonitz M. Quantum Kinetic Theory. Teubner, Stuttgart-Leipzig, 1998.

5. Kadanoff L.P., Baym G. Quantum Statistical Mechanics. Benjamin, New York, 1962.

6. Danielewicz P., Ann. Phys. (N.Y.), 1984, 152, 239.

7. Botermans W., Malfliet R., Phys. Rep., 1990, 198, 115.

8. Kremp D., Schlanges M., Bornath Th., J. Stat. Phys., 1985, 41, 661.

9. Morozov V.G., Röpke G., Ann. Phys. (N.Y.), 2009, 324, 1261.

10. Pukhov A., Rep. Prog. Phys., 2003, 66, 47.

11. Klein S., Rev. Mod. Phys., 1999, 71, 1501.

12. Santala M.I.K., et al., Phys. Rev. Lett., 2000, 84, 1459.

13. Bezzerides B., DuBois D.F., Ann. Phys. (N.Y.), 1972, 70, 10.

14. Bret A., Firpo M-C., Deutsch C., Phys, Rev. E, 2004, 70, 04641.

15. Tautz R.C., Schlickeiser R., Lerche I., J. Math. Phys., 2007, 48, 013302.

16. Špička V., Lipavský P., Phys. Rev. Lett., 1994, 73, 3439.

17. Špička V., Lipavský P., Phys, Rev. B, 1995, 52 (20), 14615.

18. Vanderheyden B., Baym G., J. Stat. Phys., 1998, 93, 843.

19. DuBois D.F. Nonequilibrium statistical mechanics of plasmas and radiation. - In: Lectures in Theoretical Physics (edited by Brittin W.E.). Gordon and Breach, New York, 1967, p. 469-619.

20. Morozov V.G., Röpke G., Condens. Matter Phys., 2006, 9, 473.

21. Mrówczyński St., Heinz U., Ann. Phys. (N.Y.), 1994, 229, 1.

22. Quack E., Henning P.A., Phys. Rev. D, 1996, 54, 3125.

23. Aurenche P., Gelis F., Kobes R., Petitgirard E., Z. Phys. C, 1997, 75, 315.

24. Aurenche P., Gelis F., Zaraket H., Phys. Rev. D, 2000, 62, 096012.

25. Köhler H.S., Malfliet R., Phys. Rev. C, 1993, 48, 1034.

26. Itzykson C., Zuber J.-B. Quantum Field Theory. McGraw-Hill, New York, 1980.

27. Ivanov Yu.B., Knoll J., Voskresensky D.N., Yad. Fiz., 2003, 66, 1950 (in Russian) [Phys. At. Nucl., 2003, 66, 1902]. 


\section{Кінетика фотонів у плазмі}

\section{В.Г. Морозов ${ }^{1}$, Г. Репке ${ }^{2}$}

1 Московський державний інститут радіоелектроніки та автоматики (Технічний університет), просп. Вернадського, 78, 119454 Москва, Росія

2 Університет Ростоку, Інститут фізики, D-18051 Росток, Німеччина

\section{Отримано 24 червня 2009 р.}

Представлено кінетичну теорію радіаційних процесів у багатосортній плазмі з релятивістськими електронами та нерелятивістськими важкими частинками. 3 використанням нерівноважних функцій Ґріна для квантової електродинаміки (КЕД) показано, що поперечні польові кореляційні функції можна природнім чином розбити на нелоренцівську частину з гострим максимумом, яка описує резонансні (пропагаторні) фотони, та поза-оболонкову складову, яка відповідає віртуальним фотонам середовища. Аналогічне розбиття отримано для поздовжніх польових кореляційних функцій та кореляційних функцій релятивістських електронів. Отримано кінетичне рівняння для резонансних фотонів зі скінченою спектральною шириною та показано, що поза-оболонкова складова кореляційних функцій частинок і полів є суттєвою для коректного розрахунку локальної потужності випромінювання в плазмі та відтворення результатів вакуумної КЕД. Обговорюється вплив плазмових ефектів на процеси випромінювання.

Ключові слова: багаточастинкова квантова електродинаміка, нерівноважні функції ґріна, релятивістська плазма

PACS: $52.20 .-j, 52.25 \mathrm{Dg}, 52.38 \mathrm{Ph}, 52.25 \mathrm{Os}, 52.27 \mathrm{Ny}$ 